Please cite this article as:

G. Landini, A. Galton, D. Randell and S. Fouad. Novel applications of discrete mereotopology to mathematical morphology, Signal Processing: Image Communication (2019), https://doi.org/10.1016/j.image.2019.04.018

Received date : 4 December 2018

Revised date : 26 March 2019

Accepted date : 22 April 2019 


\title{
Novel applications of Discrete Mereotopology to Mathematical Morphology
}

\author{
Gabriel Landini ${ }^{\mathrm{a}}$, Antony Galton ${ }^{\mathrm{b}}$, David Randell ${ }^{\mathrm{a}}$, Shereen Fouad ${ }^{\mathrm{a}, \mathrm{c}}$ \\ ${ }^{a}$ School of Dentistry, University of Birmingham, UK \\ ${ }^{b}$ Department of Computer Science, University of Exeter, UK \\ ${ }^{c}$ School of Computing, Engineering and the Built Environment, Birmingham City \\ University, UK
}

\begin{abstract}
This paper shows how the Discrete Mereotopology notions of adjacency and neighbourhood between regions can be exploited through Mathematical Morphology to accept or reject changes resulting from traditional morphological operations such as closing and opening. This leads to a set of six morphological operations (here referred to generically as minimal opening and minimal closing) where minimal changes fulfil specific spatial constraints. We also present an algorithm to compute the RCC5D and RCC8D relation sets across multiple regions resulting in a performance improvement of over three orders of magnitude over our previously published algorithm for Discrete Mereotopology.
\end{abstract}

Keywords: mathematical morphology, discrete mereotopology, image processing, spatial reasoning

\section{Introduction}

This paper centres on the processing of spatial relationships between discrete regions using Mathematical Morphology (MM). There has been a longstanding interest in formal definitions of adjacency and containment between image regions as those types of relations can form a basis for model building in image contents retrieval and analysis. This has applications to problems where the description of hierarchical structure is important, for instance, in biological imaging numerous problems revolve around the characterisation of relations of diverse nature, for instance molecules in organelles, organelles in cells, cells in tissue compartments and tissues in organs. The subject has 
been approached from a variety of points of view, including digital polygon geometry [1], digital topology [2, 3], hierarchical modelling [4] and connected filtering operators $[5,6,7]$.

Bloch $[8,9,10,11]$ has provided an extensive body of work on spatial relations in computer vision and identified ways to symbolically and programmatically harness and represent the inherent imprecision arising from image formation, post-processing, perception and the semantics related to certain spatial relationships sought. In [11] Bloch shows that MM can function as unifying framework for spatial knowledge representation and provides connections to formal logics, in particular raising the possibility of implementing Region Connection Calculus (RCC) [12] operators (as well as providing a MM definition for the RCC's tangential proper part (TPP) relation). In [9], it is proposed to construct modal logics using MM and the notion of adjunction [13] to define modal operators that can be utilised for symbolic representation and interpretation of spatial relationships. In [14] the notion of fuzzy adjacency between image objects was investigated and formally defined so the concept of adjacency can extended (e.g. using fuzzy MM formulations) to accommodate degrees of adjacency by means of admissible transformations that lead to strict adjacency and thus allow consistent representations and the management of imprecision mentioned earlier.

Research has also focused on applying MM and spatial reasoning to discrete spaces with the purpose of applying spatial reasoning to digital images. In this context, Galton [2, 3] introduced the notion of Discrete Mereotopology (DM) where he develops various mereotopological concepts for discrete spaces. Our work in $[15,16]$ shows that a subset of DM functions (closure and interior) map directly to the MM dilation and erosion operators [17] respectively, commonly used in image processing. In [2] that mapping was exploited to implement the full spatial relation set given by the RCC5D and RCC8D logics [12] in terms of MM. Briefly, the relation sets RCC5D and RCC8D encode five and eight set of relations respectively that capture various notions of parthood, overlap and contact. After mechanically verifying DM theorems adopted in the imaging algorithms (using the theorem prover SPASS [18]) we implemented the RCC5/8D relation sets and exploited several DM theorems as short-cuts in imaging algorithms to compute operations on pairs of regions. DM can therefore be used to perform certain types of segmentation and model-testing analyses based on MM procedures. Those analyses have applications in histological imaging, where segmented histological components regions of interest (those corresponding to, e.g., nuclei and 
cell bodies) represent valid theoretical models of histological reality that are related in specific ways in terms of their spatial relations $[15,16]$. This logical, model-based approach to image interpretation provides a clean formal semantic framework in which to interpret image segmentation results and, furthermore, guarantees that the imaging algorithms encoding theorems in DM are provably sound. It also enables development of algorithms that explicitly encode and 'reason' about spatial relations and local structure (e.g., cell and tissue organisation) as well as facilitating the encoding of other structural data of interest, such as the spatial localisation of molecular markers in cells and tissues.

Next we report new applications of DM that enrich MM operations. The paper is organised as follows. First, we visit the definitions of adjacency, connection and region neighbourhood in DM and their MM counterparts. Next we present a new, more efficient version of the RCC5D and RCC8D algorithm that that outlined in a previous publication [16]. Finally, we discuss a novel application of DM that extends MM with a the notions of morphological minimal closing and minimal opening, where DM is used to restrict the changes of the traditional MM closing and opening operations so the original region shape is minimally modified, while still achieving a desirable result. The paper concludes with a discussion.

\section{Methods}

The convention adopted here is that images consist of $2 \mathrm{D}$ square pixel arrays with 8-adjacency, meaning every non-boundary pixel of the array is surrounded by 8 neighbours forming a $3 \times 3$ pixel matrix. Image regions are sets of pixels locally-connected under 8-neighbour adjacency, representing objects of interest in the image. We assume that these regions exist in binary images but can include multiple planes or slices representing the same spatial reality, so that regions can share the same image space without being merged.

\subsection{Adjacency}

The adjacency relation between pixels is captured by a reflexive and symmetric relation $\mathrm{A}(x, y)$, meaning that pixel $x$ is adjacent to or equal to pixel $y$. $\mathrm{A}(x, y)$ is satisfied if $d(x, y) \leq \sqrt{2}$, where $d: \mathbb{Z}^{2} \times \mathbb{Z}^{2} \rightarrow \mathbb{R}$ is the twodimensional Euclidean distance function defined on pixel coordinates in $\mathbb{Z}^{2}$. In DM terms [15], the adjacency relation between regions $X$ and $Y$ is referred 
to as external contact and is denoted $\mathrm{EC}(X, Y)$. It is built from two other relations, namely contact: ${ }^{1}$

$$
\mathrm{C}(X, Y) \equiv_{\mathrm{def}} \exists x, y[x \in X \& y \in Y \& \mathrm{~A}(x, y)],
$$

and overlap:

$$
\mathrm{O}(X, Y) \equiv_{\text {def }} X \cap Y \neq \emptyset
$$

that is, the intersection between overlapping regions $X$ and $Y$ is non-null. External contact is then defined as:

$$
\mathrm{EC}(X, Y) \equiv_{\operatorname{def}} \mathrm{C}(X, Y) \& \neg \mathrm{O}(X, Y) .
$$

In [14] Bloch et al. showed that the adjacency relation (or external contact in DM [15]) reworked in MM is equivalent to:

$$
\mathrm{EC}(X, Y) \equiv(X \cap Y=\emptyset) \&(X \oplus B) \cap Y \neq \emptyset,
$$

where $\oplus$ represents a morphological dilation operation with a $3 \times 3$ square structuring element or kernel $B$ [17] (assumed to be centered at the orign of space to guarantee the extensivity of the dilation). Thus region $X$ has external contact with region $Y$ if the two regions do not intersect and the dilation of $X$ leads to a non-empty intersection with $Y$.

\subsection{Disconnection and region neighbourhood}

In DM, a pair of regions $X$ and $Y$ are said to be disconnected if they are not in contact, i.e., $\neg \mathrm{C}(X, Y)$; this is denoted $\mathrm{DC}(X, Y)$. This relation can also be defined in terms of the mereotopological discrete closure operation $\left(\mathrm{cl}_{\mathrm{D}}\right)$, instead of connection, as follows:

$$
\mathrm{DC}(X, Y) \equiv \mathrm{cl}_{\mathrm{D}}(X) \cap Y=\emptyset .
$$

Here the function $\mathrm{cl}_{\mathrm{D}}(X)$ is defined as the union of the set of pixels whose immediate neighbourhoods overlap $X$, where the immediate neighbourhood of a pixel $x, N(x)$, contains just those pixels which are adjacent to $x$, including pixel $x$ itself:

\footnotetext{
${ }^{1}$ The symbols $\exists, \&, \in, \cap, \neg$ and $\equiv$ are read "there exists", "and", "is a member of", "intersection", "not", and "if and only if", respectively; $\emptyset$ denotes the empty set.
} 


$$
\mathrm{cl}_{\mathrm{D}}(X)=_{\text {def }}\{x \mid \mathrm{O}(N(x), X)\} .
$$

In the case of our assumed 8-connected square grid, $\mathrm{cl}_{\mathrm{D}}(X)$ is equivalent to the dilation of $X$ using a structuring element $B$, which in our model consists of an arbitrary pixel and its immediate neighbourhood, so

$$
\mathrm{Cl}_{\mathrm{D}}(X)=X \oplus B .
$$

Therefore definition (5) translates into MM as:

$$
\mathrm{DC}(X, Y) \equiv(X \oplus B) \cap Y=\emptyset .
$$

We also define a special type of neighbourhood relation between pairs of regions that is not part of the RCC5/8D sets but is particularly useful when considering binary regions residing in the same image: region $Y$ is a neighbour of $X$ and separated from it by one pixel. We name this relation NC (for neighbourhood connection) ${ }^{2}$ and define it as:

$$
\mathrm{NC}(X, Y) \equiv_{\text {def }} \mathrm{EC}\left(\mathrm{cl}_{\mathrm{D}}(X), Y\right),
$$

which in MM terms corresponds to

$$
\mathrm{NC}(X, Y) \equiv \mathrm{EC}((X \oplus B), Y) .
$$

These formulae allow implementation of the extended MM functions that follow in Section 4. Figure 1 shows examples of the RCC8D relation set and the special cases of $\mathrm{NC}$ and $\mathrm{PO} *$.

\subsection{Region Connection Calculus via Mathematical Morphology}

In [15] we introduced equivalences between DM and MM allowing DM to be implemented and understood in terms of MM procedures. Those equivalences make it convenient to develop DM using standard image processing applications supporting basic MM operations (erosion, dilation, reconstruction). In [16], an DM algorithm implementation was presented which made use of the overlap of binary regions in images. That algorithm computes the spatial relations between two regions (self-connected or not) residing in different images. For many applications, however, it is required to find the

\footnotetext{
${ }^{2}$ In DM, the relation $\mathrm{NC}$ is symmetric, i.e., $\mathrm{NC}(X, Y) \rightarrow \mathrm{NC}(Y, X)$.
} 


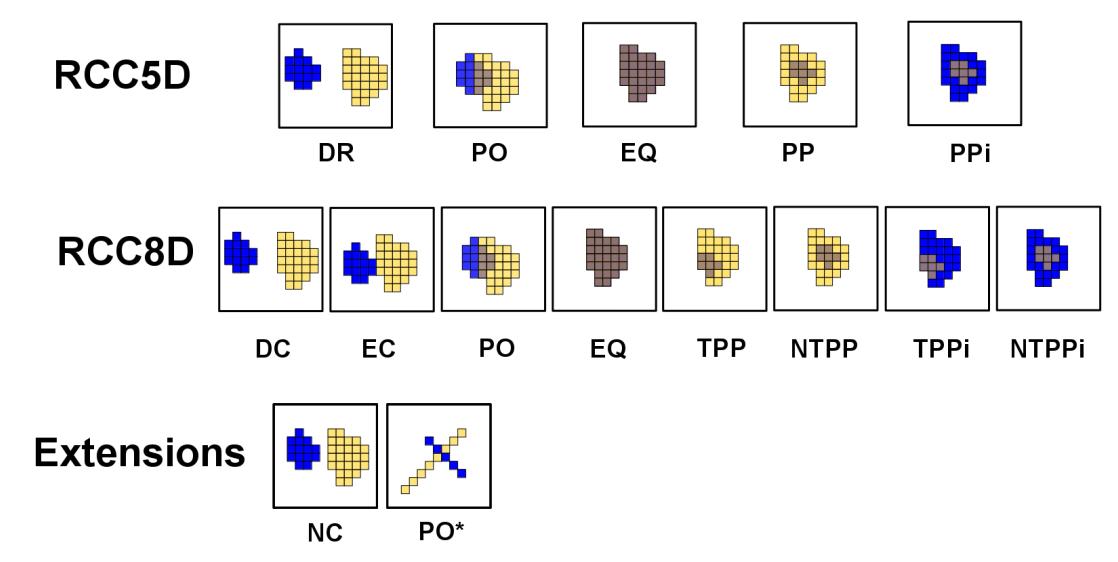

Figure 1: The five and eight spatial relations that hold between regions in the RCC5D and RCC8D sets in the discrete domain. The blue regions represent region $X$ and the yellow regions $Y$, the intersection $X \cap Y$ being shown in brown. The names in RCC5D stand for disjoint (DR), partial overlap (PO), equal (EQ), proper part (PP) and proper part inverted (PPi). The RCC8D set makes additional distinctions: disconnection (DC), external connection (EC), partial overlap (PO), tangential proper part (TPP), non-tangential proper part (NTPP). TPPi and NTPPi are the inverse relations, e.g., TPPi $(X, Y)$ means the same as TPP $(Y, X)$. The extensions considered here are NC for 'neighbourhood connection' (a case of a DC relation where the regions are one dilation away from adjacency) and PO* (a case of EC occurring on 'crossing objects' that do not share any overlapping pixels), which while possible in the discrete domain, is counter-intuitive with real-world objects.

relations held between multiple regions ${ }^{3}$ contained in pairs of images (e.g., biological objects across different confocal microscopy imaging planes, or stain channels). In such cases, the computation can be decomposed into a sequence of analyses between pairs of self-connected regions: first extract two given regions into new empty images (maintaining their relative positions), next compute the relation held between them using the said algorithm, and repeat this for all remaining region pairs. That implementation exploits the 'start pixels' of regions (the first pixel in a given region encountered in raster scan order) and uses morphological reconstruction [19] to extract each region separately and apply the RCC test to the extracted pair. Such an approach, however, quickly becomes computationally expensive; when dealing with ei-

\footnotetext{
${ }^{3}$ While a non-null region in DM is simply the union of an arbitrary set of pixels, the algorithmic manipulation of regions being assumed here is typically restricted to connected components, or simple regions.
} 
ther large images (for which morphological reconstruction is slow) or images featuring many regions (the number of tests is given by the product of the number of regions across the images, complexity $\mathrm{O}(\mathrm{nm}))$. Some shortcuts have been identified, for instance in RCC5D, the disjoint relation DR can be assumed by default for all region pairs and other spatial relations only computed in cases of overlap, avoiding a considerable number of tests. Similarly, $E Q$ can be identified in those regions pairs whose minimum pixel value is 3 in the sum of image $X$ (labelled as 0 and 1) and image $Y$ (labelled as 0 and 2). However, the distribution of DM relations varies with the image content and therefore such shortcuts do not necessarily lead to noticeable execution time improvements. The next section presents a more efficient algorithm which avoids the decomposition of the computation into an exhaustive sequence of region pairs. The procedure shows a considerable advantage in execution time compared to our previous algorithm 2 and it enables DM analysis to be more efficient and therefore applicable to high-throughput workflows.

\section{Fast RCC5/8 Algorithm}

We assume $n$ binary regions in image $X$ and $m$ binary regions in image $Y$. The aim is to identify the spatial relations of the regions in $X$ with the regions in $Y$. Those relations can be stored in an $n \times m$ matrix, here called the 'RCC table' (stored as an image) where the $x$ and $y$ coordinates are indices pointing to the $x$ th and $y$ th regions in $X$ and $Y$ respectively.

\subsection{Computing RCC5D}

First, two images are generated using connected component-labelling, one where all regions in $X$ have unique labels (according to their raster scan order) and the other similarly with the labels of the regions in $Y$. We call these images $X_{\text {labelled }}$ and $Y_{\text {labelled }}$ respectively. Two additional images are computed, one where pixels belonging to regions in $X$ are labelled as 1 (or foreground) and 0 otherwise (background) and the other where pixels belonging to regions in $Y$ are labelled as 2 (foreground) and 0 otherwise. These two images are summed to produce a third image $X Y$, where pixels now have values of 1 (the pixel is in $X$ but not $Y$ ), 2 (it is in $Y$ but not $X$ ), 3 (a region of $X$ overlaps a region of $Y$ at that location) or 0 (image background). A further binary image $O$ is computed as the intersection (overlap) of $X$ and $Y$. These overlaps arise in the case of RCC5D relations PO, EQ, PP and 
PPi. Inspection of the values of the pixels of the overlaps in $O$ (by redirecting to $X_{\text {labelled }}$ and $Y_{\text {labelled }}$ ) reveals which two regions form a given overlap. We store the label values of the regions of $O$ in arrays ovX [ ] and ovY [ ]. The regions in images $X$ and $Y$ involved in overlapping relations are also inspected by redirection to image $X Y$, and their minimum pixel values are stored in arrays $\operatorname{minX}[]$ and $\operatorname{minY}[]$. These arrays store information on whether a given region contains non-overlapping pixel values of 1 or 2 (which occur in $\mathrm{PPi}$ and $\mathrm{PO}$ cases) or whether all the pixels in a region are overlapping (value 3, which occurs in PP and EQ cases). Adding minX and minY provides enough information to compute four of the five RCC5D relations (i.e., all those that involve region overlaps)

\begin{tabular}{lccc}
\hline Relation & minX & minY & minX+minY \\
\hline $\operatorname{PO}(x, y)$ & 1 & 2 & 3 \\
$\mathrm{EQ}(x, y)$ & 3 & 3 & 6 \\
$\operatorname{PP}(x, y)$ & 3 & 2 & 5 \\
$\operatorname{PPi}(x, y)$ & 1 & 3 & 4 \\
\hline
\end{tabular}

Table 1: Minimum values of pixel composition of overlapping regions $X$ and $Y$. Region labels are: background $=0, X=1, Y=2, X \cap Y=3$. The columns minX and minY indicate the minimum value in regions $X$ and $Y$ respectively when a given relation holds.

From this scheme, it can be worked out that the relation $R$ between regions $X_{\mathrm{ovx}[\mathrm{i}]}$ and $Y_{\mathrm{ov}[\mathrm{i}]}$ in images $\mathrm{X}$ and $\mathrm{Y}$ (given by the overlap region $O_{i}$ ) is:

$$
R[i]=\operatorname{out}[\operatorname{minX}[\operatorname{ovX}[\mathrm{i}]]+\operatorname{minY}[\mathrm{ovY}[\mathrm{i}]]],
$$

where out [ ] is a look-up table holding labels for relations $\mathrm{PO}=3, \mathrm{EQ}=6$, $\mathrm{PP}=5$ and $\mathrm{PPi}=4$ (see Table 1 , rightmost column). Since the only remaining RCC5D relation, DR, does not involve an overlap, DR can conveniently be assumed by default for all possible region pairs and during the analysis the values in the RCC table are only updated for those regions involved in overlapping relations using the procedure described. The procedure is shown in pseudocode in Algorithm 1.

\subsection{From $R C C 5 D$ to $R C C 8 D$}

RCC8D introduces the notion of contact between regions, covering both overlap and adjacency [1] and resulting in eight spatial relations which pro- 
Algorithm 1 Pseudocode for RCC5D computation across multiple regions in images $X$ and $Y$.

1. Default all relations between regions in $X$ and $Y$ to DR.

2. Compute labelled images $X_{\text {labelled }}$ and $Y_{\text {labelled }}$ where each region has a unique label.

3. Compute image $X Y$, coded as $1 \rightarrow$ pixel of a region in $X$ but not $Y$, $2 \rightarrow$ pixel of a region in $Y$ but not $X, 3 \rightarrow$ pixel of a region in both $X$ and $Y$.

4. Compute binary image $O$, coded as $0 \rightarrow$ background, $1 \rightarrow X \cap Y$.

5. Create arrays ovX [ ] and ovY [ ] holding the information of which regions in $X$ and $Y$ form overlaps in $O$, by inspecting region labels in $X_{\text {labelled }}$ and $Y_{\text {labelled }}$.

6. Create arrays $\operatorname{minX}[$ ] and $\operatorname{minY}[$ ] by inspecting for each region in $O$ the minimum pixel value for that region in image $X Y$.

7. For each region in $O, \operatorname{minX}+\operatorname{minY}$ gives the RCC5D relation: $3 \rightarrow$ $\mathrm{PO}, 4 \rightarrow \mathrm{PPi}, 5 \rightarrow \mathrm{PP}, 6 \rightarrow \mathrm{EQ}$. 
vide a more fine-grained spatial description than RCC5D. The RCC5D relation DR is split into the RCC8D relations EC (external connection) and DC (disconnection), the RCC5D relation PP is split into TPP and NTPP (tangential and non-tangential proper part respectively), the former occurring when the proper part abuts the background regions, the latter when it does not; the same thing happens, mutatis mutandis, with the inverse relations. The RCC5D results obtained by the method described earlier can be reprocessed to capture the $\mathrm{RCC} 8 \mathrm{D}$ relations of the same set of regions by performing single forward image scans testing for adjacency patterns (rather than processing region-pairs one at a time). The computation of RCC8D could be seen as a decomposition of the problem into a set of sub-problems (first compute RCC5D, then re-process the image without having to consider all region pairs, while exploiting the previously obtained results), similar to the type of problem reduction sought in dynamic programming [20]. We search for the presence/absence of certain patterns of adjacent pixels occupancy which, in conjunction with the known RCC5D relations, are indicative of specific RCC8D relations. The cases PO and EQ are the same in RCC5D and RCC8D. Of the remaining cases, suppose that we know the RCC5D relation between regions $X$ and $Y$ is DR. Then the RCC8D relation can only be either DC or EC. For it to be EC there must be at least one instance where a pixel of $X$ is adjacent to a pixel of $Y$. The relation is DC is assumed by default and then we scan the image looking for the adjacency pattern; if it is found, EC is returned, if the pattern is not found, then the default DC holds good.

The following notation is used to describe the two-pixel patterns. Consider a pixel $p$ and let $n$ be one of its immediate neighbours. Set $p(X)$ to be 1 or 0 according as $p$ does or does not belong to region $X$; and likewise with $p(Y), n(X)$, and $n(Y)$. Then the two-pixel pattern exhibited by the pair $p, n$ with respect to $X$ and $Y$ is denoted by the quadruple $(p(X), p(Y), n(X), n(Y))$.

From the above, we can say that a DR relation between $X$ and $Y$ will be DC unless one of the quadruple patterns $(0,1,1,0)$ or $(1,0,0,1)$ is exhibited for some $p, n$ pair in the image, in which case the relation is EC. Similarly, a case of $\operatorname{PP}(X, Y)$ will be $\operatorname{NTPP}(X, Y)$ unless patterns $(0,0,1,1)$ or $(1,1,0,0)$ occur, in which case it will be $\operatorname{TPP}(X, Y)$; and likewise with $\mathrm{PPi}, \mathrm{NTPPi}$, and TPPi. To perform these tests, the image is scanned using the 'forward mask' of pixel $p$, shown in Figure 2 .

At each $p$ we determine the two-pixel patterns formed by $p$ with each 


\begin{tabular}{|l|l|l|}
\hline$N_{(x-1, y-1)}$ & $N_{(x, y-1)}$ & $N_{(x+1, y-1)}$ \\
\hline$N_{(x-1, y)}$ & $P_{(x, y)}$ & $N_{(x+1, y)}$ \\
\hline$N_{(x-1, y-1)}$ & $N_{(x, y+1)}$ & $N_{(x+1, y+1)}$ \\
\hline
\end{tabular}

Figure 2: The forward mask of pixel $p$. The pixel patterns for occupancy of regions in image $X$ and $Y$ are tested between the central pixel $p$ in the neighbours $n$ in the 'forward mask' (shaded pixels). The pixels in the 'backward mask' do not need to be tested because the patterns have already been visited during the raster scan.

of the shaded elements of the mask in turn. As the scan progresses, an accumulator records whether these patterns have arisen, and the relabelling of the region relations is done after the scan is finished. The form of the mask is dictated by the fact that the image is scanned top-to-bottom and left-to-right (no need to look at the pattern for, e.g., $p=(x, y), n=(x, y-1)$, since this will already have been detected when $(x, y-1)$ played the role of $p$, with the pattern $p=(x, y-1), n=(x, y))$.

\subsection{Extended relations $\mathrm{NC}$ and $\mathrm{PO} *$}

The NC relation (definition 9) describes two regions separated by a onepixel gap (Figure 1). This occurs when a region is detected as DC and the pixel patterns over the next-nearest neighbours (the external shell of a $5 \times 5$ neighbourhood) show that pixels $p$ (in the neighbourhood centre) and $n$ (in the shell) are occupied by pixels of regions of $X$ and $Y$, or $Y$ and $X$, respectively. The $\mathrm{PO} *$ relation arises when two 8-connected regions 'cross' each other in corner-connected regions, without overlapping or sharing any pixels (Figure 1). Such a pattern can commonly arise in the square lattice and it is interpreted as EC in RCC8D. In practical applications, however such results can be unintuitive (e.g. a linear object crosses another without ever "passing through" it) and it might be useful to identify these occurrences. This is done by inspecting $2 \times 2 n$ and $p$ pixel patterns for exclusive cornerconnected pixel pairs in relations that have been identified as EC. 


\subsection{Complexity analysis and performance}

The old algorithm in [16] uses morphological binary reconstruction to extract every pair of regions before calculating the relational model held between them. It has been shown in [21] that morphological reconstruction is a computationally expensive, highly non-linear procedure. Its complexity depends on the number of component/pixels to be reconstructed. Even for the efficient/best-compromise algorithms [22] it is recognised that a mean case complexity analysis would be extremely difficult to compute because of the variety of input images that may be used. In addition to utilizing reconstruction, the computational complexity of the core of the old algorithm (in the worst case scenario) is quadratic, $\mathrm{O}(\mathrm{nm})$ (when $\mathrm{m} \approx \mathrm{n}$ ) because the relations are computed between all possible region pairs ( $\mathrm{n}$ and $\mathrm{m}$ ) one at a time, or subquadratic when $\mathrm{m} \neq \mathrm{n}$. While some shortcuts were identified (e.g. to avoid computing relations between those regions that are further away than two dilations, guaranteed to be DC), an important bottleneck remains with the binary reconstruction steps necessary to extract the region pairs.

The new Algorithm 1, first, avoids extracting individual pairs of regions into new images to compute the relations, thus avoiding morphological reconstruction altogether. Secondly, it computes the RCC5D relation from a sequence of steps that reduce the complexity from quadratic to linear yielding to an average case complexity of $(\mathrm{O}(\mathrm{n}+\mathrm{m}))$. In particular, steps 1,3 and 4 in Algorithm 1 have a constant-time algorithm of order $1(\mathrm{O}(1))$. Step 2 (image labelling) requires a maximum time complexity of $\mathrm{O}(\mathrm{n}+\mathrm{m})$. Steps 5,6 and 7 process the overlapping subregions that occur across the two images. It should be noted that relations PP, PPi and EQ are one to one, and result in one overlapping segment per region pair. A worst case scenario where all the relations held are any of the above (therefore $n=m$ ) would lead to a scaling of these steps to $\mathrm{O}(\mathrm{n})$ which is still less than $\mathrm{O}(\mathrm{n}+\mathrm{m})$. The $\mathrm{PO}$ relation, however, is a special case in the sense that a region can have more than one overlapping subregion (with one or multiple other regions). For instance large and convoluted regions could potentially lead to a scaling higher than $\mathrm{O}(\mathrm{n})$. While is not possible to foresee what regions configurations may be found in segmented images, it is nevertheless possible to clarify the impact of this unknown, experimentally. In a series of performance tests on random binary images (detailed below) we found that on average, the number of overlapping subregions across 500 tests (average 7152, maximum 19431 regions) was smaller than the number of regions $n+m$ (average 11168, maximum 38934). The running time of the proposed algorithm would there- 
fore, on average, increase linearly with the total number of regions $\mathrm{O}(\mathrm{n}+\mathrm{m})$, with some exceptional configurations where it could be higher depending of the number and nature of the PO relations. As examined experimentally, situations where this is above the quadratic complexity of the old algorithm appear to be unlikely. The successive forward passes on the labelled images to compute the RCC8D relation set, as well as the extended relations NC and $\mathrm{PO} *$, are of the order $\mathrm{O}(1)$ and therefore do not increase the algorithm complexity.

Figure 3 shows the difference in performance, in seconds, of the previously published [16] and the new algorithms on $512 \times 512$ pixels, random binary images with varying probabilities, $\mathrm{p}$, of foreground pixels. The tests were performed on the ImageJ platform, version 1.51 [23] under the Linux operating system on an Intel Xeon CPU (E31225) at 3.1GHz. The plot shows the average of 5 runs at each $\mathrm{p}$ in steps of 0.01 . The average difference over all $\mathrm{p}$ was an improvement of 491 times faster than the previous algorithm, while largest difference was found at $\mathrm{p}=0.42$ where the new algorithm was 1684 times faster than the old one. The execution times appear to be dependent not only on the number of regions per image but also on the proportion of the different types of relations that occur at various $\mathrm{p}$ (not shown). A slight advantage was noticed for the old algorithm implementation on images with the highest $\mathrm{p}$, (where only very few regions exist, the images being mostly occupied by one large region), but this difference, in practical terms, becomes negligible as the execution times in those cases are all at a fraction of a second.

\section{New morphological filters: Minimal closing and opening}

In addition to the applications of DM in histological imaging $[15,16$, 24], the fast algorithm enables new MM operators with reasonable speed performance to be designed, exploiting the relations between image regions and the changes they undergo after other morphological operations.

In MM, the operation closing $\phi$ with a kernel $B$ is defined as the dilation of a region, followed by an erosion [17]:

$$
\phi_{B}(X)=_{\text {def }}(X \oplus B) \ominus B .
$$

Closing is an extensive transformation, where voids in regions, and details that cannot contain the translations of kernel $B$, are filled. Note that 


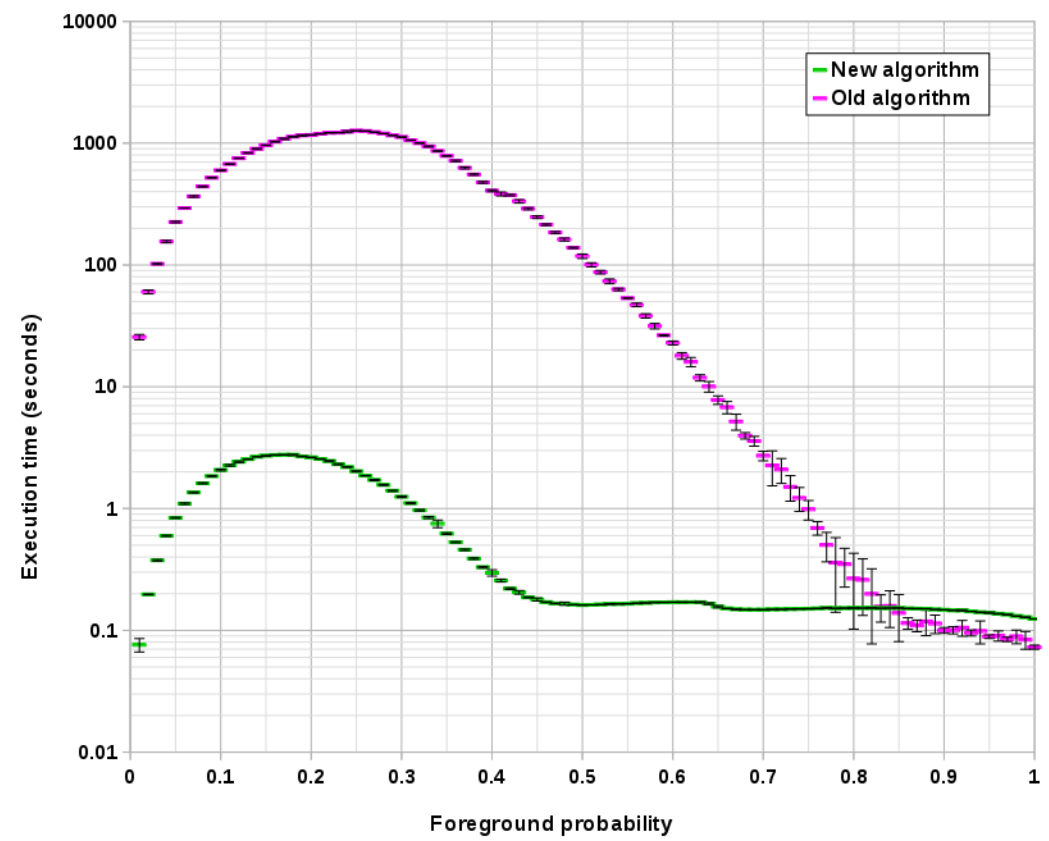

Figure 3: Differences between the average execution times of the old and new RCC8D algorithms. The tests were done, on random binary $512 \times 512$ pixel images with varying foreground pixel probabilities. Each point is the average of 5 runs and the vertical bars indicate one standard deviation from the mean.

mereotopological closure, which refers to a topological operator defined on a discrete space, does not correspond to closing but to dilation in MM, despite the similarity in their names. When closing binary regions, voids are filled with the foreground value. While the rest of this section only deals with closings, there is a dual MM operation with respect to the set complement, namely opening, an anti-extensive transform, which instead of filling, removes those object pixels that cannot be fully covered by the translations of kernel $B$.

Closing is commonly used to 'fill in' gaps between nearby regions desired to be joined, to fill small holes in regions and to reduce the complexity of region boundaries ('shorelines' from now on). These actions are, however, not independent; gaps, holes and shoreline irregularities are processed concurrently as the operation does not differentiate between them. In certain circumstances, however, it might be desirable to achieve only one of those results, e.g., joining nearby regions while avoiding major modifications of the 
shoreline details that do not yield a connection to another region. Using DM this can be modelled as follows. In MM, the set of pixels added to the original region by the closing operator is known as the black top-hat):

$$
\operatorname{BTH}(X)=\phi_{B}(X)-X .
$$

The black top-hat often consists of a set of disconnected components, the elements of which we call black top-hat segments. In DM this is defined immediately following, where $\mathrm{BTH}_{\text {seg }}(Y, X)$ is read as ' $Y$ is a connected component of the black top-hat of $X^{\prime}$ and $\mathrm{BTH}_{\text {segs }}(X)$ is the set of black top-hat segments of $\mathrm{X}$. The relation $\mathrm{CC}(Y, X)$ which we use here, read as ' $Y$ is a connected component of $X^{\prime}$, is also defined in DM-see [15], p 572:

$$
\begin{gathered}
\mathrm{BTH}_{\mathrm{seg}}(Y, X) \equiv_{\text {def }} \quad \mathrm{CC}(Y, \mathrm{BTH}(X)), \\
\mathrm{BTH}_{\mathrm{segs}}(Y, X)=_{\text {def }}\{Y \mid \mathrm{CC}(Y, \mathrm{BTH}(X))\} .
\end{gathered}
$$

The set-builder notation used to define function $\mathrm{BTH}_{\text {segs }}(X)$ in definition 15 returns a set of regions, namely the $Y$ s. In DM, however, where a region rather than a set of regions is required as the output of a function ${ }^{4}$, the set union operator is added, i.e.,

$$
\mathrm{BTH}_{\mathrm{segs}}(X)=\bigcup\{Y \mid \mathrm{CC}(Y, \mathrm{BTH}(X))\},
$$

and the same principle applies for equations 19, 20 and 25. The relation between $Y$ and $X$, given $\mathrm{BTH}_{\text {seg }}(Y, X)$, is always $\mathrm{EC}$, that is to say they are adjacent (externally connected) regions - remembering here that the segments are the result of an extensive transformation. In addition, the segments could also be adjacent to other regions in their neighbourhood; if the distance $\epsilon$ separating pairs of regions is no more than half the width of kernel $B$, the black top-hat segments create 'bridges' between originally disconnected (i.e., DC) regions. When considering region $\mathrm{X}$ and all other regions $\mathrm{Y}$ in the segmented image, two black top-hat segment types can arise. First we have what we call shorelines where $\mathrm{BTH}_{\text {shoreline }}(Y, X)$ is read as ' $\mathrm{Y}$ is a connected shoreline component of the black top-hat of $X$ '. In this case the black top-hat segment $\mathrm{Y}$ adjoins exactly one connected component of $\mathrm{X}$ :

\footnotetext{
${ }^{4}$ Remembering that in DM, a region can comprise several disjoint, region-parts as well as being a simple region.
} 


$$
\begin{aligned}
& \mathrm{BTH}_{\text {shoreline }}(Y, X) \equiv_{\text {def }} \\
& \qquad \mathrm{CC}(Y, \mathrm{BTH}(X)) \& \exists Z[\forall U[\mathrm{CC}(U, X) \& \mathrm{EC}(U, Y)] \leftrightarrow U=Z],
\end{aligned}
$$

i.e., $Y$ is a connected shoreline component of the black top-hat of $X$ if and only if $Y$ is a connected component of the black top-hat of $X$, and there exists exactly one connected component of $X$ that is EC to $Y$.

The second case is where we have a black top-hat segment that forms a bridge between two regions. $\mathrm{BTH}_{\text {bridge }}(Y, X)$ is read as " $Y$ is a black top-hat bridge of $X^{\prime \prime}$ :

$$
\begin{aligned}
& \mathrm{BTH}_{\text {bridge }}(Y, X) \equiv_{\text {def }} \mathrm{CC}(Y, \mathrm{BTH}(X)) \& \\
& \quad \exists Z, U[\mathrm{CC}(Z, X) \& \mathrm{CC}(U, X) \& Z \neq U \& \mathrm{EC}(Z, Y) \& \mathrm{EC}(U, Y)],
\end{aligned}
$$

which is similar to definition (17) except there are now at least two connected components of $X$ externally connected to the black top-hat segment $Y$ of $X$, not one.

The spatial relations that hold between the black top-hat segments and the original regions provides a means for identifying those which act as bridges (between DC region pairs) and those which do not (and consequently only modify a region shoreline). From this it follows that the black top-hat segments adjacent to only one region are shoreline modifiers (including hole filling, when the holes can be filled by the kernel), and those adjacent to more than one region are bridges. Retaining one or another type, (e.g., by means of binary reconstruction [19]), gives rise to two types of conditional minimal closing, shoreline smoothing without region merging:

$$
\phi_{B}^{\text {shoreline }}(X)=\left\{Y \mid \mathrm{BTH}_{\text {shoreline }}(Y, X)\right\},
$$

and region merging without boundary smoothing:

$$
\phi_{B}^{\text {bridge }}(X)=\left\{Y \mid \mathrm{BTH}_{\text {bridge }}(Y, X)\right\} .
$$

Note that in $\mathrm{RCC} 8 \mathrm{D}$, the notion of shoreline or boundary of a region does not differentiate between the 'outside' boundary and the boundary with an internal hole. The DM treatment of region holes is dealt with later in this paper. 
With regards to implementation, the different black top-hat variants are sorted by an exhaustive analysis of the relations between all original regions versus all black top-hat segments generated after an MM closing (i.e., $X \oplus$ $B) \ominus B)$ ). Those results, arranged in an $m \times n$ matrix or table indexed by regions and black top-hat segments in scan order (here named the RCC table), provide a convenient way to search for those special relations. The DM relation between a given region and a black top-hat segment can be one of two, out of the eight possible outcomes of the RCC8D region set: either DC or EC. To identify 'bridge' black top-hat segments, we use indexing of the original regions and black top-hat segments in the $x$ and $y$ axis of the RCC table respectively: the number of EC instances in a row indicates the number of different regions a given black top-hat segment is adjacent to. Black top-hat segments with total EC counts per row equal to 1 are therefore shoreline modifiers (i.e., they are adjacent to only one region), while those instances with counts exceeding 1 are guaranteed to be bridges. As will be seen comparing Figures $4 \mathrm{f}$ and $4 \mathrm{~g}$, black top-hat shoreline segments include those completely surrounded by a region; we call these segments lakes. In DM this can be defined as follows, where $\mathrm{BTH}_{\text {lake }}(Y, X)$ is read as ' $\mathrm{Y}$ is a black top-hat lake of $\mathrm{X}^{\prime}$; the definition uses the DM definition of a hole defined later:

$$
\mathrm{BTH}_{\text {lake }}(Y, X) \equiv_{\text {def }} \mathrm{BTH}_{\text {seg }}(Y, X) \& \operatorname{Hole}(Y, X) .
$$

The crucial distinction between a shoreline and lake black top-hat segment of a given region is that a lake also satisfies what it is to be a hole in that region which again is encoded in another RCC table indexing regions and holes. Examples of binary region merging with minimal shoreline smoothing and shoreline smoothing without region merging are given in Figure 4.

While black top-hat segments have the same connectivity as the original regions (e.g., 8-connected) the minimal closing can be minimised further by considering only the adjacency relations of their 4-connected subcomponents. The rationale for this is that retaining a given black top-hat segment is similar to adding some background pixels to the foreground. Since the 8-connected foreground convention implies a 4-connected background, it is possible to restrict minimal closing to the 4-connected sub-components of a given black top-hat segment that satisfies the bridge or shoreline properties described earlier and not including the whole black top-hat region. Figure 5 shows the effect of retaining such 4-connected components in cases of pro- 


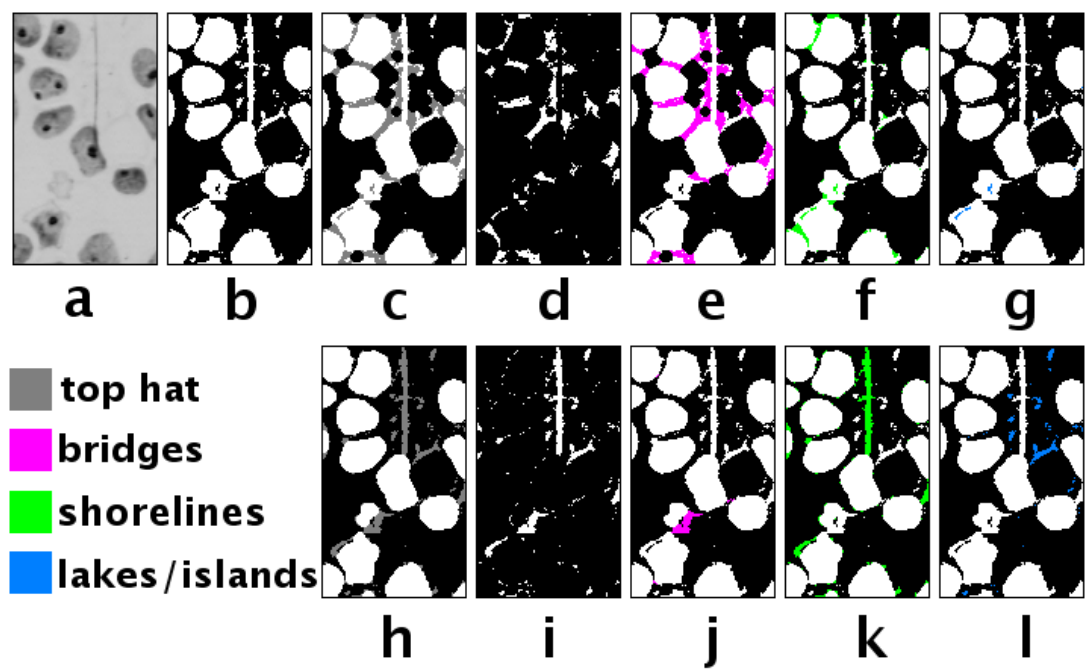

Figure 4: Closing and minimal closing of binary images with a disc of radius 3. The original greyscale image of lymphocytes stained with silver nitrate for detection of nucleolar organising regions (dark spots)(a) was segmented with the minimum error thresholding algorithm [25] (b). In (c) the traditional closing (with the added pixels in grey that make the 'black top-hat' (d). Panel (e) shows in magenta the black top-hat segments that have an adjacency relation with more than one region in (b), acting as bridges. We call this operation 'minimal closing bridges'. Panel (f) shows those black top-hat segments that have adjacency to only one other region in (b) (minimal closing shorelines), while in (g) are shown the lakes which are black top-hat segments that have no connection to the rest of the background's subset that intersects the image boundaries. Panel (h) shows the traditional opening (with added pixels in grey that make the 'white top-hat' (i). Panels (j-1) shown the minimal opening of bridges, shorelines and islands respectively.

cessing regions with null interior.

Finally, the dual operation of the closing is opening, $\Upsilon$ :

$$
\Upsilon_{B}(X)=_{\text {def }}(X \ominus B) \oplus B,
$$

and the corresponding top-hat transformation for the opening is called white top-hat:

$$
\operatorname{WTH}(X)=_{\operatorname{def}} X-\Upsilon_{B}(X),
$$

which identifies the segments that were removed from the original after the opening operation. As before and mirroring definitions for the black top-hat we define a white top-hat segment $Y$ of region $X$, and the set of white top-hat segments of $X$ : 


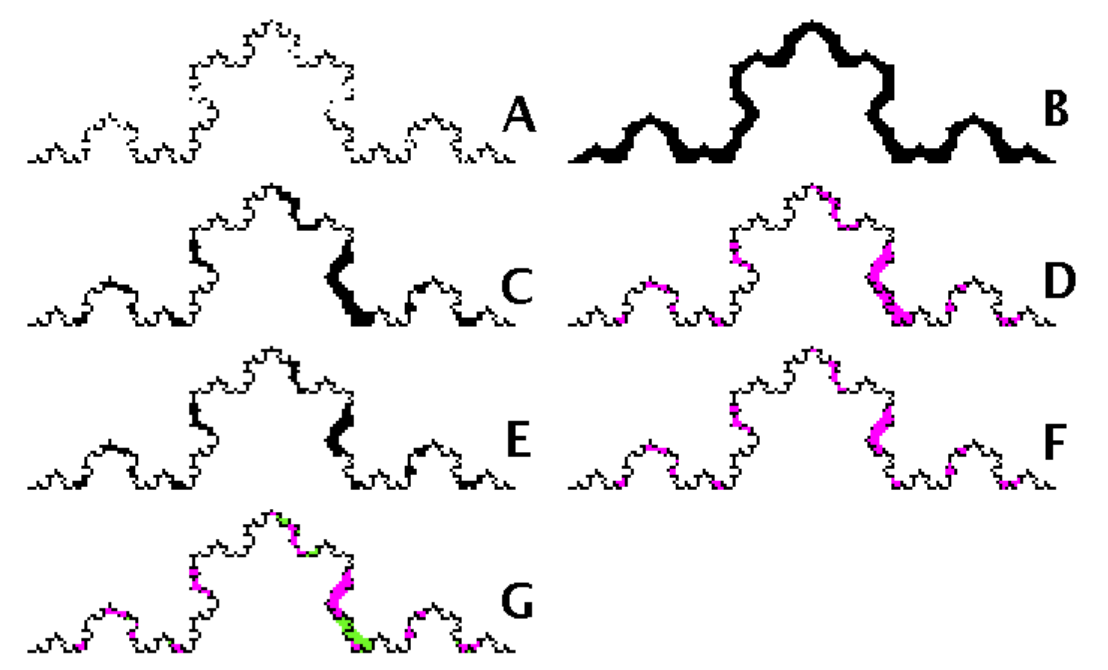

Figure 5: Closing versus minimal closing. (a) A digitised version of a 4th order von Koch curve with discontinuities, resulting in 30 fragments. The target is to merge all pieces into a single region. (b) The classical result using morphological closing with a circular kernel of radius 4 (the smallest kernel that closes all gaps). Note the loss of detail in the result. (c) A minimal closing where the gaps between any two fragments were filled independently with the smallest kernel sizes possible until a single region was obtained. (d) The detail of the minimal closing (black is the original set, magenta (dark grey in B/W version) represents the black top-hat segments. (e) and (f) show the same example, but this time retaining only the 4-connected subregions of each black top-hat segment $\left(\mathrm{BTH}_{\text {segs }}\right.$ in the text) that acts as a mereotopological bridge between fragments. Note that this closing modifies the original even less than in (c). (g) shows in green (bright grey) the part sub-regions of the black top-hat segments that were not necessary to retain to achieve the minimal closing, i.e., the difference between (d) and (f).

$$
\begin{gathered}
\mathrm{WTH}_{\text {seg }}(Y, X) \equiv_{\text {def }} \mathrm{CC}(Y, \mathrm{WTH}(X)), \\
\mathrm{WTH}_{\text {segs }}(X)=\{Y \mid \mathrm{CC}(Y, \operatorname{WTH}(X))\} .
\end{gathered}
$$

It is therefore possible to implement minimal opening operations as the dual of minimal closing. Note that while opening is an anti-extensive transformation, the white top-hat segments are in relation EC to the regions in the opened image, that is: $\mathrm{WTH}_{\text {seg }}(Y, X) \rightarrow \mathrm{EC}\left(Y, \Upsilon_{B}(X)\right)$. The two new dual minimal opening operations are open shorelines and open bridges, depending on which type of white top-hat segments are retained or removed. It is also possible to define an additional minimal opening operation that removes 
those white top-hat segments that are DC to all other regions in the opened image. We call this procedure opening islands, and its dual, closing lakes. Interestingly, these opening islands and closing lakes are equivalent to opening and closing by reconstruction, respectively [26]. This sequence of morphological operations combining MM with the explicit relations of DM shows the potential for defining a variety of fine-grained morphological operators that target a particular goal. It also highlights the importance of securing computationally efficient ways to compute and store relations between pairs of regions when processing segmented images such as those assumed in the RCC table, where these relations are explicitly used in these new operators. An example of the advantage of these new operators is shown in Figure 5. Here connecting fragments in a discontinued curve can be restricted to places where the closing leads to fragment connections, without interference at locations where the connection is not necessary. By so doing we preserve the original as much as possible with a less dramatic loss of global detail than traditional closing.

Similarly to minimal binary opening and closing, the procedures above can directly be applied to process greyscale images via threshold decomposition (although the threshold decomposition it is usually an inefficient procedure). Figure 6 shows examples of the greyscale versions of the minimal closing and opening respectively.

\section{Discussion}

Bloch $[11,9]$ originally suggested that $\mathrm{RCC}$ relations can be defined in MM, and specifically provides the translation forthe $T P P(X, Y)$ relation [11], which is equivalent to ours in [15]. It should be noted that while MM is not specific about discrete or continuous space, that is not an exact translation of the RCC8 TPP relation on discrete space, because RCC presupposes an infinitely divisible one. Instead, for the case of discrete space, the connections drawn are with the RCC8D relation set of discrete mereotopology.

The implementation of RCC5D, RCC8D and additional DM relations as MM procedures opens a range of new opportunities to extend some operations beyond their original design by means of exploiting spatial relations held between regions. This is specially useful when designing analytical procedures that can benefit from mechanically reasoning about image contents.

The approach presented here allows the results of closing and openings to be made conditional on certain types of modifications which might not 


\section{Closing \\ Opening}

Original

\section{Closing Opening}

Close bridges

\section{Closing Opening}

Open bridges

\section{Closing Opening}

Close boundaries

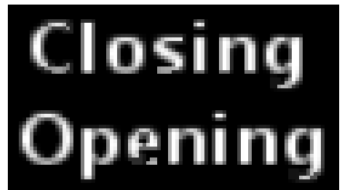

Open boundaries

\section{Closing Opening}

\author{
Close lakes
}

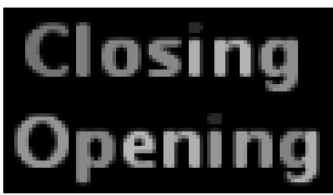

Open islands

Figure 6: Greyscale minimal closing and opening. The examples were computed using a $3 \times 3$ kernel on a greyscale image of text. Note (second row) how minimal closing bridges connect nearby regions without modifying the shorelines or filling lakes and how the closing of lakes does not affect the shoreline features. The open bridges procedure leads to fragmentation of regions in the original without affecting other shoreline features (compared to open shorelines), while the opening of islands removes the white top-hat segments with no adjacency relations to any other patterns at a given grey level.

be straightforward to achieve otherwise or might require more complex approaches such as multiscale operators and directional information [27]. While conditional filtering is not new, traditional conditional morphological operators apply their constraints in a given local sub-image (given by the kernel). To replicate this type of filtering region-wise is challenging because classical methods require additional processing to account for relations between regions and conditions on these to be met, whereas in DM it is built into its very foundations.

The bridges, boundaries, island and lakes regions in relation to opening and closing (i.e. the white top-hat and black top-hat segments) have similarities to what Soille and Vogt call 'binary patterns' [28] for which they identified formulae for their computation (and include some additional patterns: core, perforations, branches and loops). For minimal closing and opening, 
however DM has the advantage of being able to relate, via the RCC table, which original regions are adjacent to those segments and therefore open the possibility to control algorithmically whether segments are included or removed from particular configurations of regions. That would would require further computation in the approach presented in [28].

There has been interest in other types of conditional operations, for example homotopic sequential filtering to preserve the topology of an image $[29,30]$ or multiscale top-hat transforms to improve image contrast [31]. Here we described how processing can be applied to changes of regions or across regions. A number of new uses for DM via MM has been recently identified in applications that require dealing with models where image regions fulfil specific spatial relation between their parts [16, 24, 32]. Such models commonly arise in histological imagery, where detected regions represent regions with special biological meanings (such as cells, nuclei, tissues, organs) that not only can be distinctly detected, but also exist in specific spatial relations and hierarchies. Such relations need to be fulfilled if the extraction of biologically relevant information from images is to be related to a given context in terms of ontological levels of organisation [33]. On a different kind of application, Cointepas [34] proposed the use of MM combined with adjacency relations to construct homotopically deformable cellular models and resolve complex problems, such as 3D cerebral cortex segmentation, where topology preservation is essential to yield not only accurate but anatomically plausible results.

The procedures presented here stem from our work in histological imaging using digital images of 2D tissue sections, and as as such are based on a 2D Cartesian grid representation. It would be desirable to further develop these concepts and algorithms in n-dimensions so they can be applied to e.g. temporal, volumetric and higher dimensional data sets. Furthermore, alternative schemes such as simplicial complexes (used to represent multidimensional data) [35], graphs [36] and hypergraphs [37, 38] (for non-lattice implementations of MM) might be advantageous for such generalisation to higher dimensions.

\section{Acknowledgements}

The research reported in this paper was supported by the Engineering and Physical Sciences Research Council (EPSRC), UK through funding under grant EP/M023869/1 'Novel context-based segmentation algorithms for 
intelligent microscopy'.

[1] A. Rosenfeld, R. Klette. Degree of adjacency or surroundedness. Technical Report, Center for Automation Research, University of Maryland, CAR-TR-53, 1984.

[2] A. Galton, The mereotopology of discrete space, in: C. Freska, D.M. Mark (Eds.), Spatial Information Theory: Cognitive and Computational Foundations of Geographic Science, 1999, 251-266.

[3] A. Galton, Discrete Mereotopology. In: Calosi C., Graziani P. (eds) Mereology and the Sciences. Synthese Library (Studies in Epistemology, Logic, Methodology, and Philosophy of Science), vol 371. Springer, Cham, 2014, 293-321.

[4] A. E. Gelfand. Hierarchical modeling for spatial data problems. Spatial Statistics 1 (2012) 30-39.

[5] H. J. A. M. Heijmans. Connected morphological operators for binary images. Computer Vision and Image Understanding 73 (1999) 99-120.

[6] J. Serra. Connections for sets and functions. Fundamenta Informaticae 41 (2000) 147-186.

[7] G. K. Ouzonis, and M. H. F. Wilkinson. Mask-based second generation connectivity and attribute filters. IEEE Transactions on Pattern Analysis and Machine Intelligence 29 (2007) 990-1004.

[8] I. Bloch. Fuzz relative position between objects in image processing: a morphological approach. IEEE Transactions on Pattern Analysis and Machine Intelligence 21 (1999) 657-664.

[9] I. Bloch. Modal logics based on mathematical morphology for qualitative spatial reasoning. Journal of Applied Non-Classical Logics 12 (2002), 399-423.

[10] I. Bloch. Mathematical morphology on bipolar fuzzy sets: General algebraic framework. International Journal of Approximate Reasoning 53 (2012) 1031-1060. 
[11] I. Bloch, Spatial reasoning under imprecision using fuzzy set theory, formal logics and mathematical morphology, International Journal of Approximate Reasoning 41 (2006) 77-95.

[12] D. A. Randell, Z. Cui, and A. G. Cohn, A spatial logic based on regions and connection. Proceedings of the Third International Conference on Knowledge Representation and Reasoning, 1992, 165-176.

[13] I. Bloch. Duality vs. adjunction for fuzzy mathematical morphology and general form of fuzzy erosions and dilations. Fuzzy Sets and Systems 160 (2009) 1858-1867.

[14] I. Bloch, H. Maître, and M. Anvari. Fuzzy adjacency between image objects. International Journal of Uncertainty, Fuzziness and KnowledgeBased Systems 5 (1997) 615-653.

[15] D. A. Randell, G. Landini, and A. Galton. A, Discrete mereotopology for spatial reasoning in automated histological image analysis, IEEE Transactions on Pattern Analysis and Machine Intelligence 35 (2013) 568-581.

[16] G. Landini, D. A. Randell, and A. Galton, Discrete mereotopology in histological imaging, in: E. Claridge, A.D. Palmer, W.T.E. Pitkeathly (Eds.), Proceedings of the 17th Conference on Medical Image Understanding and Analysis, 2013, 101-106.

[17] J. Serra, Image Analysis and Mathematical Morphology, vol. 1, Academic Press, 1982.

[18] C. Weidenbach, D. Dimova, A. Fietzke, R. Kumar, M. Suda, and P. Wischnewski, SPASS Version 3.5, Proceedings of the 22nd International Conference on Automated Deduction, 2009, 140-145.

[19] L. Vincent, Morphological grayscale reconstruction in image analysis: Applications and efficient algorithms, IEEE Transactions on Image Processing, 2 (1993) 176-201.

[20] P. F. Felzenszwabl, and R. Zabih. Dynamic Programming and Graph Algorithms in Computer Vision. IEEE Transaction on Pattern Analysis and Machine Intelligence 33 (2011) 721-740. 
[21] P. Balázs. Complexity results for reconstructing binary images with disjoint components from horizontal and vertical projections, Discrete Applied Mathematics 161 (2013) 2224-2235.

[22] L. Vincent. Morphological grayscale reconstruction in image analysis: Efficient algorithms and applications. Technical Report 91-16, Harvard Robotics Laboratory, 1991.

[23] W. S. Rasband, ImageJ, U. S. National Institutes of Health, Bethesda, Maryland, USA, https://imagej.nih.gov/ij/, 1997-2018 (accessed 23 April 2018).

[24] D. A. Randell, A. Galton, S. Fouad, H. Mehanna, and G. Landini. Mereotopological correction of segmentation errors in histological imaging. Journal of Imaging 3 (2017) 63.

[25] J. Kittler, and J. Illingworth. Minimum error thresholding, Pattern Recognition 19 (1986) 41-47.

[26] P. Soille. Morphological Image Analysis: Principles and applications, second edition, Springer, 2004, ch. 6.

[27] M. A. Oliveira, and N. J. Leite. A multiscale directional operator and morphological tools for reconnecting broken ridges in fingerprint images, Pattern Recognition 41 (2008) 367-377.

[28] P. Soille, and P. Vogt. Morphological segmentation of binary patterns, Pattern Recognition Letters 30 (2009) 456-459.

[29] M. Couprie, and G. Bertrand. Topology preserving alternating sequential filter for smoothing 2D and 3D objects. Journal of Electronic Imaging, Society of Photo-optical Instrumentation Engineers 13 (2004) 720730 .

[30] R. Mahmoudi, and M. Akil. Enhanced computation method of topological smoothing on shared memory parallel machines. EURASIP Journal on Image and Video Processing (2011) 16.

[31] J. C. M. Román, H. L. Ayala, and J. L. V. Noguera. Top-hat transform for enhancement of aerial thermal images, 30th SIBGRAPI Conference on Graphics, Patterns and Images (SIBGRAPI), Niteroi, 2017, 277-284. 
[32] H. Strange, Z. Chen, E.R.E. Denton, and R. Zwiggelaar. Modelling mammographic microcalcification clusters using persistent mereotopology. Pattern Recognition Letters 47 (2014) 157-163.

[33] A. Galton, G. Landini, D. Randell, and S. Fouad. Ontological levels in histological imaging, in: R. Ferrario, W. Kuhn W (Eds.) Formal Ontology in Information Systems, 2016, 271-284.

[34] Y. Cointepas. Modélisation homotopique et segmentation 3D du cortex cérébral à partir d'IRM pour la résolution des problèmes directs et inverses en EEG et en MEG. PhD Thesis. Télécom ParisTech, 1999.

[35] F. A. Salve Dias. A study of some morphological operators in simplicial complex spaces. PhD Thesis, Université Paris-Est, 2012.

[36] J. Cousty, L. Najman, F. Dias, and J. Serra. Morphological filtering in graphs, Computer Vision and Image Understanding 117 (2013) 350-385.

[37] I. Bloch, and A. Bretto. Mathematical morphology on hypergraphs: preliminary definitions and results. In: I. Debled-Rennesson, E. Domenjoud, B. Kerautret, P. Even (Eds.), Discrete Geometry for Computer Imagery, Lecture Notes in Computer Science, vol. 6607, Springer, Berlin, Heidelberg, 2011, 429-440

[38] I. Bloch. Morphological Links Between Formal Concepts and Hypergraphs. International Symposium on Mathematical Morphology and its Applications to Signal and Image Processing, 2017, Springer, Cham, 16-27. 